\title{
Foliations and global inversion
}

\author{
Eduardo Cabral Balreira
}

\begin{abstract}
We consider topological conditions under which a locally invertible map admits a global inverse. Our main theorem states that a local diffeomorphism $f: M \rightarrow \mathbb{R}^{n}$ is bijective if and only if $H_{n-1}(M)=0$ and the pre-image of every affine hyperplane is non-empty and acyclic. The proof is based on some geometric constructions involving foliations and tools from intersection theory. This topological result generalizes in finite dimensions the classical analytic theorem of Hadamard-Plastock, including its recent improvement by Nollet-Xavier. The main theorem also relates to a conjecture of the aforementioned authors, involving the well-known Jacobian conjecture in algebraic geometry.
\end{abstract}

Mathematics Subject Classification (2000). Primary 58K15; Secondary 57R30.

Keywords. Jacobian conjecture, Hadamard's theorem, global inversion theorem.

\section{Introduction}

In this paper we are concerned with the problem of finding topological conditions ensuring that a local diffeomorphism is bijective. A classical result in this direction is the well-known Hadamard-Plastock Theorem (see [5] and [11]). It states that a Banach space local diffeomorphism $f: X \rightarrow X$ is bijective provided

$$
\inf _{x \in X}\left\|D f(x)^{-1}\right\|^{-1}>0 .
$$

The proof of the Hadamard-Plastock Theorem follows from simple arguments involving covering spaces. In recent years new topological and geometric ideas have been introduced in the subject of global invertibility, pushing the field in different directions (see, for instance, [1], [6], [7], [8], [9], [12], [15], [18], [20], and [21]). The emerging picture reveals that global invertibility is also influenced by more subtle topological phenomena. In [7], Nollet and Xavier established a substantial improvement to the Hadamard-Plastock Theorem when $\operatorname{dim} X<\infty$. Using degree theory, they showed in [7] that a local diffeomorphism $f: \mathbb{R}^{n} \rightarrow \mathbb{R}^{n}$ is bijective if there exists a complete Riemannian metric $g$ on $\mathbb{R}^{n}$ such that

$$
\inf _{x \in \mathbb{R}^{n}}\left\|D f(x)^{*} v\right\|_{g}>0 \quad \text { for all } v \in S^{n-1} .
$$


Notice that (1.2) is an improvement of (1.1) since

$$
\left\|D f(x)^{-1}\right\|^{-1}=\left\|D f(x)^{*-1}\right\|^{-1}=\inf _{|v|=1}\left\|D f(x)^{*} v\right\| .
$$

Furthermore, it is easy to produce examples that satisfy (1.2) but not (1.1). Arguments from elementary Morse theory (see [10, p. 112]) show that if (1.2) holds, then the pre-images of affine hyperplanes $H$ must satisfy $f^{-1}(H) \times \mathbb{R} \cong \mathbb{R}^{n}$ (note that $\left.D f(x)^{*} v=\nabla\langle f(x), v\rangle\right)$. In particular, by the Künneth formula, $f^{-1}(H)$ is acyclic (recall that a topological space is called acyclic if it has the homology of a point).

In this paper we show that the above mentioned analytical results are but a manifestation of a topological phenomenon.

Theorem 1.1. A local diffeomorphism $f: \mathbb{R}^{n} \rightarrow \mathbb{R}^{n}$ is bijective if and only if the pre-image of every affine hyperplane is non-empty and acyclic.

In Section 3 we will point out a connection between the above theorem and the Jacobian conjecture in algebraic geometry. The non-trivial half of Theorem 1.1 consists in establishing injectivity and surjectivity. Its proof is based on some geometric constructions involving foliations, and the computation of intersection numbers of certain chain complexes. Theorem 1.1 also allows for an analytic corollary that is stronger than the results in [7], in the sense that one can choose the metric to suit the unit vector $v$.

\section{Preliminaries}

Given a compact smooth manifold $M^{n}$ and a finite cover, we would like to have a systematic way to describe the intersections of the sets in the cover. Likewise, once a point is given we want to describe exactly all the sets in the cover that contain the given point. To this end, we will consider a triangulation of $M$ and view the top dimensional cells as the sets of the covering. We set our notation as follows. Denote by $T(M)$ a triangulation on $M$ (whose existence is guaranteed by [17]) and let $e(k)_{j}$ be the $j^{\text {th }} k$-cell of $T(M)$. The set of indexes of $k$-cells will be denoted by $E(k) \subset \mathbb{N}$. Also, given a triangulation $T(M)$, let $T_{k}(M)$ be the $k$-skeleton of $M$. Whenever the context is clear, we will refer to the triangulated space simply as $M$.

This combinatorial approach allow us to easily address the properties we mentioned above. For instance, given a simplex $e(k)_{j}$, the star of $e(k)_{j}$ describes all the simplexes that contain $e(k)_{j}$. In our results, we will be interested in finding all the $(k+1)$-simplexes that contain $e(k)_{j}$. This is easily accomplished by looking at the vertices of the link of $e(k)_{j}$, denoted by $\operatorname{Lk}\left(e(k)_{j}\right)$.

We now review the basic definitions from intersection theory. We define in $M^{n}$ the intersection number $(\bmod 2)$ between $A^{p}$ a $p$-cycle and $B^{q}$ a $q$-cycle, where $p+q=n$ 
by \# $\left(A^{p}, B^{q}\right)$. We note that when $A^{p}, B^{q}$ represent transverse submanifolds, then $\#\left(A^{k}, B^{n-k}\right)$ represents the number of geometric intersections mod 2 . The property that we highlight is that intersection number depends only on the homology class. For details and formal definitions we refer the reader to [14].

Finally, we can also define linking numbers between cycles. Let $X^{p}$ and $Y^{q-1}$ be two nonintersecting cycles in $\mathbb{R}^{n}$ with $p+q=n$. For $Z^{p+1}$ a bounding chain of $X^{p}$, i.e. $\partial Z^{p+1}=X^{p}$, we define the linking number between $X^{p}$ and $Y^{q-1}$ as

$$
\operatorname{Lk}\left(X^{p}, Y^{q-1}\right)=\#\left(Z^{p+1}, Y^{q-1}\right),
$$

which is independent of the choice of the bounding chain of $X^{p}$.

\section{Injectivity}

Let us consider a local diffeomorphism $f: M \rightarrow \mathbb{R}^{n}$, where $M$ is a smooth connected manifold. Our goal is to understand under which topological conditions the map $f$ is injective. There is a conceptual link between injectivity and connectedness. For instance, it is clear that a locally invertible map is injective if and only if the pre-image of every 0-dimensional affine subspace (i.e., a point) is connected (possibly empty). An analogous statement can be made if one goes one dimension higher and considers lines instead of points, that is, a locally invertible map is injective if the pre-image of every line is connected.

In view of these observations, Nollet and Xavier [7] made the following conjecture.

Conjecture 3.1. A local diffeomorphism $f: \mathbb{R}^{n} \rightarrow \mathbb{R}^{n}$ is injective if the pre-images of every affine hyperplane is connected (possibly empty).

At the present time this conjecture remains open and its significance is better seen in Algebraic Geometry where it would provide a positive answer for the Jacobian conjecture (recall that the Jacobian conjecture states that a polynomial local biholomorphism $F: \mathbb{C}^{n} \rightarrow \mathbb{C}^{n}$ is invertible, see [3], [16]). Indeed, if $F: \mathbb{C}^{n} \rightarrow \mathbb{C}^{n}$ is a polynomial local biholomorphism, and $H \subset \mathbb{C}^{n}$ is a real hypersurface foliated by complex hyperplanes $V$, then by a Bertini type theorem $F^{-1}(V)$ is connected for a generic $V$ (see [13], Corollary 1 of Theorem 3.7). From this one can easily check that $F^{-1}(H)$ is connected and hence one would establish the Jacobian conjecture.

The result below establishes a weaker version of the Nollet-Xavier conjecture, where connectedness is replaced by acyclicity.

Theorem 3.2. A local diffeomorphism $f: \mathbb{R}^{n} \rightarrow \mathbb{R}^{n}$ is injective if the pre-image of every affine hyperplane is either empty or acyclic. 
In fact, we observe that we may weaken the hypotheses of Theorem 3.2 to obtain the following stronger result. We say that an affine hyperplane $H \subset \mathbb{R}^{n}$ is parallel to a line $\ell$ in $\mathbb{R}^{n}$ provided that $\ell \cap H=\emptyset$ or $\ell \subset H$.

Theorem 3.3. For $n \geq 3$, let $f: M \rightarrow \mathbb{R}^{n}$ be a local diffeomorphism where $M$ is a (necessarily non-compact) connected manifold with $H_{n-1}(M)=0$. If there exists a line $\ell$ in $\mathbb{R}^{n}$ such that the pre-image of every affine hyperplane parallel to $\ell$ is either empty or acyclic, then $f$ is injective.

The proof of Theorem 3.3 is based on geometric constructions of chain complexes, the computation of the intersection number between these objects, and the maximal lift of lines. Since the computation of intersection numbers is done with objects belonging to the domain of $f$, we need to require the extra assumption on the homology of $M$. Observe that the cases in Theorem 3.3 when $n=1,2$ are trivially true without any extra assumptions on $M$.

We stress that in our arguments we will only require the existence of local lifts. In fact, by the Hadamard-Plastock Theorem [11], if all lines admit global lifts the map is already bijective. We refer to a local lift of a line $\ell=\left\{t w \mid w \in \mathbb{R}^{n}, t \in \mathbb{R}\right\}$ with respect to $f$ as a path $\alpha:(-\varepsilon, \varepsilon) \rightarrow M, \varepsilon>0$ such that $f(\alpha(t))=t w$. Observe that by the Inverse Function Theorem, if $f$ is a diffeomorphism a local lift of a line always exists in the above sense. We say that $\alpha:(-\delta, \delta) \rightarrow M$ is the maximal lift of $\ell$ if $\delta=\sup \{\varepsilon \mid \alpha$ admits a local lift for $\varepsilon>0\}$. Furthermore, $\ell$ has a global lift if its maximal lift satisfies $\delta=\infty$. Finally, what is important for us is the fact that the maximal lift of a line is properly embedded in the domain. In our notation, $\alpha$ is properly embedded if it leaves every compact set of $M$ as $|t|$ increases to $\delta$.

3.1. Beginning of the proof of Theorem 3.3. Assume that there is a point $p$ in the image of $f$ with at least two distinct points $q_{0}$ and $q_{1}$ in its pre-image. Since translations do not change any of the hypotheses, we assume for simplicity that $p=0$. Our goal is to construct a $(n-1)$-cycle $\Gamma^{n-1}$ so that the intersection number of $\Gamma^{n-1}$ with the maximal lift of the line $\ell$ passing through the origin will necessarily be zero, as the maximal lift is properly embedded. A simple argument will then show that $f$ must have a critical point along the lift, thus establishing the desired contradiction.

First, we give an outline of the proof. Consider $\varepsilon>0$ so that the ball $V=$ $B(0 ; \varepsilon) \subset f(M)$ has diffeomorphic pre-images $U_{0}$ and $U_{1}$ around $q_{0}$ and $q_{1}$, respectively. Next, let $Y$ be the $(n-1)$-equatorial disk of $V$ determined by $\ell$, that is, the intersection of the orthogonal hyperplane to $\ell$ and $V$. The cycle $\Gamma^{n-1}$ we seek will be constructed to resemble a topological cylinder that connects the induced equatorial disks of $U_{0}$ to $U_{1}$, denoted by $X_{0}$ and $X_{1}$, respectively. We construct $\Gamma^{n-1}$ as follows. Take a hyperplane parallel to $\ell$ which intersects $\partial V$ tangentially at $v$ and is denoted by $\mathcal{H}_{v}$. As we change the hyperplane $\mathcal{H}_{v}$ by moving it around $\partial V$, the pre-images 
$u_{i} \in \partial U_{i}$ of $v$, for $i=0,1$, can be continuously connected by paths in $f^{-1}\left(\mathcal{H}_{v}\right)$, at least for nearby hyperplanes. In this way we construct small lateral pieces of $\Gamma^{n-1}$.

One then tries to put together all those local data. In so doing, one is forced to consider the situation where, for a fixed hyperplane $\mathcal{H}_{v}$, there are multiply-defined paths joining the same pre-images of points in $f^{-1}\left(\mathcal{H}_{v}\right)$. Whenever this occurs, the topological hypotheses that $f^{-1}\left(\mathcal{H}_{v}\right)$ is acyclic will be used to fill in the gaps. See Figure 3.1 for a depiction of this process when $n=3$.
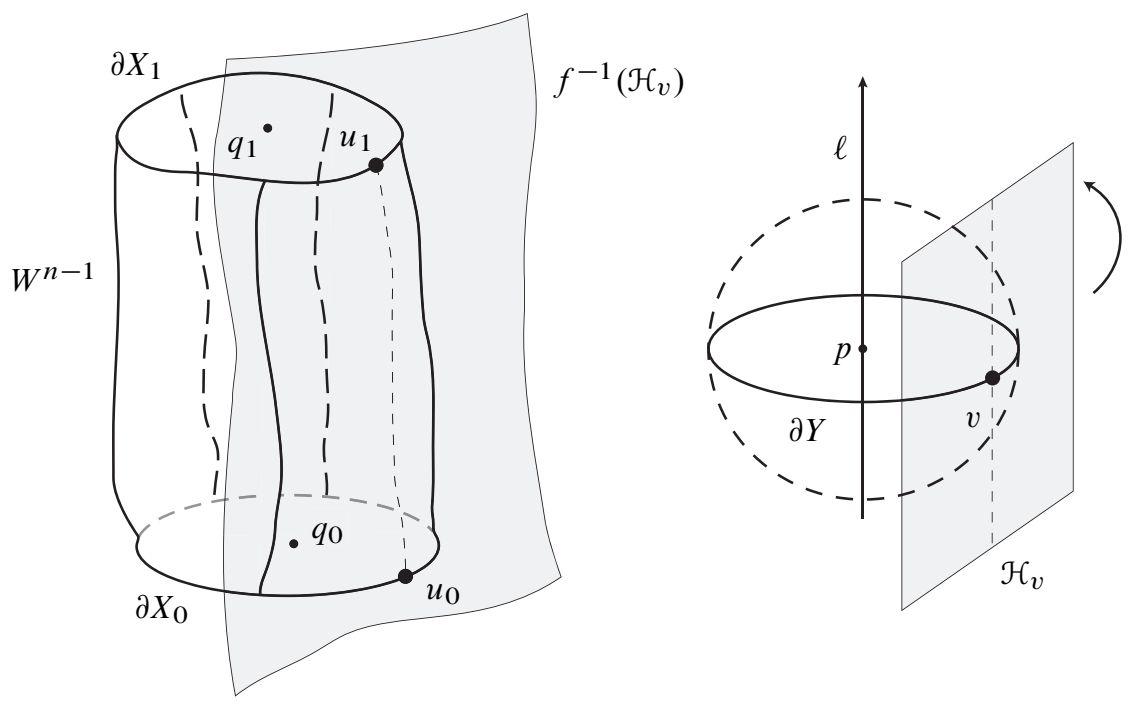

Figure 3.1. Construction of paths connecting $\partial X_{0}$ to $\partial X_{1}$ by the revolution of affine hyperplanes.

In order to determine how the lateral pieces will fit together and how such gaps should be filled, we consider a combinatorial decomposition of $\partial Y$ in terms of a triangulation. Here we observe that $\partial Y \cong S^{n-2}$, so such triangulation always exist. The process of putting together the pieces of $\Gamma^{n-1}$ will be done in steps according to the dimension of the carrier of each point. More precisely, first we consider a point and the $(n-2)$-cells it may possibly belong and construct chain complexes that correspond to the lateral pieces indicated above. Next, points that belong to the lower dimensional skeleton of $\partial Y$ will be consider more than once in the initial step. Hence, in the following step we consider the $(n-3)$-skeleton of $\partial Y$ and determine the bounding chains according to the higher dimensional cells that contain it. We repeat this process until we consider the 0 -skeleton of $\partial Y$. The existence and properties of $\Gamma^{n-1}$ are established in the following lemma.

Lemma 3.4. There exists a geometric (singular) chain complex $\Gamma^{n-1} \in S_{n-1}(M)$ that may be represented as $\Gamma^{n-1}=X_{0}+W^{n-1}+X_{1}$ such that $W^{n-1}$ is a chain 
complex with $\partial W=\partial X_{0}+\partial X_{1}$ and for all $q \in \operatorname{supp} W^{n-1}$ (in its image in $M$ ), there exists $v \in \partial Y$ so that $q \in f^{-1}\left(\mathcal{H}_{v}\right)$.

The proof of Lemma 3.4 follows the outline above where we will construct all the singular chain complexes of $W^{n-1}$ and the attaching maps. This argument uses ideas from combinatorial topology and we postpone it until next section. We proceed to establish Theorem 3.3, but first we remark that we are interested in the existence of a geometric intersection (i.e., number of points in the set theoretical intersection) between $\Gamma^{n-1}$ and the maximal lift of $\ell$. Therefore we consider intersection numbers and homology with $\mathbb{Z}_{2}$ coefficients, thus avoiding heavier notational concerns regarding orientation and leaving the proof simpler and more geometric.

Assuming $\Gamma^{n-1}$ is constructed as in Lemma 3.4, we compute the intersection number of $\Gamma^{n-1}$ and the maximal lift of $\ell$ starting at $q_{0}$ which we denote by $\gamma$. We claim that $\gamma$ must intersect $\Gamma^{n-1}$ in another point besides $q_{0}$ and we will show that it is $q_{1}$. First, we see that $\gamma$ is properly embedded in $M$. Indeed, if we decompose $\gamma$ as $\gamma_{-} \wedge \gamma_{+}$as the maximal lift of $\ell$ in the negative and positive direction, respectively, starting at $q_{0}$. It is then clear that $\gamma_{-}$and $\gamma_{+}$are not entirely contained in any compact subset of $M$, otherwise the lift would not be maximal.

Now, the fact that $\Gamma^{n-1} \in S_{n-1}(M)$, i.e., a cycle and $H_{n-1}(M)=0$ implies that there exists a bounding singular chain $\Sigma^{n}$, with $\partial \Sigma^{n}=\Gamma^{n-1}$ and a compact set $K$ so that $\Sigma^{n} \subset K$. Thus $\Gamma^{n-1}$ is a representative of the trivial element in $H_{n-1}(M, M-K)$. We also have that $\gamma \in H_{1}(M, M-K)$ and from the fact that intersection numbers depend only on the homology class, we have

$$
\#\left(\Gamma^{n-1}, \gamma\right)=0
$$

Indeed, $H_{n-1}(M, M-K)=H_{n-1}(M / M-K)$ and since $\Sigma^{n} \subset K$, we have $\Gamma^{n-1} \sim 0$ in $H_{n-1}(M / M-K)$ as well.

From Lemma 3.4 and by definition of intersection numbers, we can write (3.1) as follows:

$$
\begin{aligned}
& 0=\#\left(\Gamma^{n-1}, \gamma\right) \\
& =\#\left(X_{0}, \gamma\right)+\#\left(W^{n-1}, \gamma\right)+\#\left(X_{1}, \gamma\right) \\
& =1+0 \quad+\#\left(X_{1}, \gamma\right) \text {, }
\end{aligned}
$$

where the first term is 1 since $f$ is a local diffeomorphism and the images of $\gamma$ and $X_{i}$ are orthogonal and the second term is zero since $\gamma \cap W^{n-1}=\emptyset$. Therefore, it must be that $\#\left(X_{1}, \gamma\right)=1$. In particular, $\gamma \cap X_{1} \neq \emptyset$ and by the choice of $\varepsilon$, it must be that $\gamma \cap X_{1}=\left\{q_{1}\right\}$. For a geometric depiction see Figure 3.2.

Finally let $\alpha \subset f^{-1}(\ell)$ be the path segment from $q_{0}$ to $q_{1}$. The image of $\alpha$ is a loop in $\ell$ that has a point $\hat{p} \in \ell \cap f(M)$ that is furthest from $p$. Now it is clear that $f$ fails to be locally invertible at the corresponding pre-image of $\hat{p}$, giving us the desired contradiction. Therefore $f$ must be injective. 

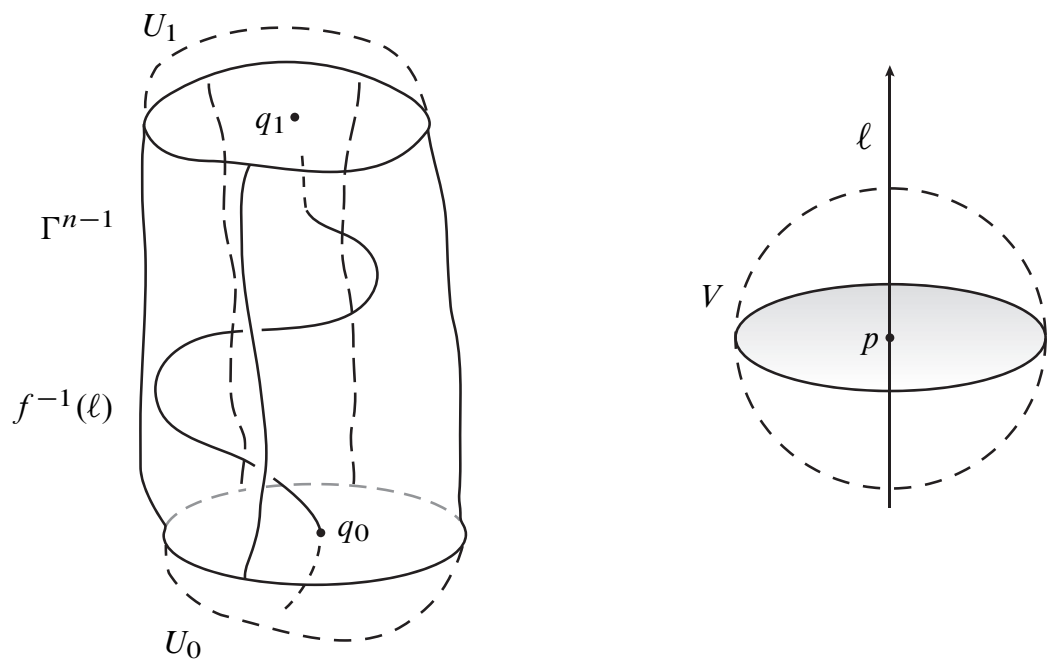

Figure 3.2. Construction of a closed chain complex $\Gamma^{n-1}$ by revolving affine hyperplanes.

3.2. Reassemblage of hyperplanes and a chain complex construction. We now establish Lemma 3.4, needed to complete the proof of Theorem 3.3. While outlining the construction of $\Gamma^{n-1}$ earlier, we encountered a key problem which simply put is attributed to the lack of uniqueness on the choice of the path used to connect the pre-images of a point in $\partial Y$. In our construction this is reflected as follows: although each path may be defined continuously within a neighborhood of a fixed point, as we consider the intersection of two neighborhoods there will possibly be two choices of paths. We claim that whenever ambiguity occurs, we may use the hypotheses of acyclicity of the pre-images of hyperplanes to define chain complexes to circumvent this problem.

We do this by considering a triangulation of $\partial Y$ with sufficiently small mesh to be determined during the proof. Heuristically, we view a neighborhood of a generic point as the top dimensional cell containing it and the triangulation will provide a way to keep track of the intersection of the multiple neighborhoods. Let $e(k)_{j}$ be the cells of such triangulation, where $k=0, \ldots, n-2$ denotes the dimension of each cell and $j \in E(k) \subset \mathbb{N}$ is the indexing set of the $k$-cells. From the initial choice of $\varepsilon>0$, we may also define an induced triangulation via the local diffeomorphism on $\partial X_{0}$ and $\partial X_{1}$ with cells $e(k)_{j}^{0}$ and $e(k)_{j}^{1}$, respectively.

We construct $W^{n-1}$ in $n-1$ steps which we enumerate from 0 to $n-2$. In step $k$ we consider points in the $(n-2-k)$-skeleton of $\partial Y$, denoted by $\partial Y_{(n-2-k)}$, and show that the possibly multiply defined chains are obtained by looking at all the higher dimensional cells containing such points and that these chains give rise to a cycle. 
Then by using the acyclicity hypotheses, we have that such cycle can be realized as the boundary of another chain complex which will be the building blocks of $W^{n-1}$.

Step 0 . The initial process is analogous to what has been outlined before, but to establish our notation we provide the formal argument. Given the initial triangulation of $\partial Y$, take $v \in e(n-2)_{\ell}$ and let $u_{i}$ be the pre-image of $v$ in $\partial X_{i}$ for $i=0,1$. From the connectedness hypotheses of $f^{-1}\left(\mathcal{H}_{v}\right)$, there is a path $W_{\ell}^{1}(v) \subset f^{-1}\left(\mathcal{H}_{v}\right)$ joining $u_{0}$ to $u_{1}$, that is, $W_{\ell}^{1}(v)$ is a 1-chain with $\partial W_{\ell}^{1}(v)=u_{0}+u_{1}$.

Next, we can continuously modify $W_{\ell}^{1}(v)$ for all points in a neighborhood of $v \in \partial Y$. This follows because $W_{\ell}^{1}(v)$ is compact and $f$ is a local diffeomorphism. By repeating this construction for every point in $\partial Y$, we obtain a cover of $\partial Y$ from which we extract a finite subcover as $\partial Y$ is compact. Then take finitely many barycentric subdivisions of $\partial Y$ until its mesh is smaller than the minimum diameter of the subcover.

Finally we redo the assignment of $W_{\ell}^{1}(v)$ for each $v \in e(n-2)_{\ell}$ using the newly obtained triangulation. This has the property that for each $(n-2)$-cell we may define a $(n-1)$-chain complex denoted by $W_{\ell}^{1} \times e(n-2)_{\ell}$ from the continuous family of paths for each $\ell \in E(n-3)$.

Step 1. In this next step, we consider points in the $(n-3)$-skeleton of $\partial Y$ as these are the points which we possibly assigned two different 1-chain complexes in the previous step. For $v \in e(n-3)_{\ell}$, we may identify all the $(n-2)$-cells that contain $e(n-3)_{\ell}$ by looking at the vertices of $\operatorname{Lk}(e(n-3) \ell)$. In this case, we have precisely two points as $e(n-3)_{\ell}$ belongs to exactly two top dimensional cells say, $e(n-2)_{1}$ and $e(n-2)_{2}$. From the previous step, we constructed two possibly distinct chain complexes $W_{1}^{1}(v)$ and $W_{2}^{1}(v)$ contained $f^{-1}\left(\mathcal{H}_{v}\right)$ joining $u_{0}$ to $u_{1}$. If it is the case they are already the same, we are done. Otherwise, consider the 1-chain $U_{\ell}^{1}(v)=W_{1}^{1}(v)+W_{2}^{1}(v)$. We claim $U_{\ell}^{1}(v)$ is a cycle. Indeed, $\partial U_{\ell}^{1}(v)=\partial W_{1}^{1}(v)+\partial W_{2}^{1}(v)=u_{0}+u_{1}+u_{0}+u_{1}=$ 0 , since we are using $\mathbb{Z}_{2}$-coefficients. From the hypotheses that $f^{-1}\left(\mathcal{H}_{v}\right)$ is acyclic, we have that $U_{\ell}^{1}(v)$ is the boundary of a 2-chain denoted by $W_{\ell}^{2}(v)$.

Now, using the fact that $f$ is a local diffeomorphism and $W_{\ell}^{2}(v)$ is compact, we can continuously define $W_{\ell}^{2}(u)$ for all $u$ in a neighborhood of $v$ in $\partial Y_{(n-3)}$. Note that in this step we are only considering points in the $(n-3)$-skeleton. Therefore we obtain a cover of $\partial Y_{(n-3)}$ which by compactness we extract a finite subcover. Next, we iterate finitely many barycentric subdivisions of the triangulation on $\partial Y$ until its mesh is smaller than the minimum diameter of the subcover. We then redo the construction of the chain complexes up to this point in steps 0 and 1 using the new triangulation. We do this so the 2-chain complex defined above can be continuously assigned for each point within a $(n-3)$-cell and we obtain a $(n-1)$-cell denoted by $W_{\ell}^{2} \times e(n-3)_{\ell}$ for each $\ell \in E(n-3)$.

Step $k$. For a generic step $k(1<k \leq n-2)$, we consider points in the $(n-2-k)$ skeleton of $\partial Y$. For $v \in e(n-2-k)_{\ell}$, we look at the $(n-1-k)$-cells that contain 
$e(n-2-k)_{\ell}$. This is the case because in the previous step $k-1$, we have defined $k$-chains $W_{i}^{k}(v)$ over $v$ these cells that $v$ belong, for some $i$. A systematic way to consider these cells is to look at the vertices of $\operatorname{Lk}(e(n-2-k) \ell)$. Let us assume that those are $e(n-1-k)_{1}, e(n-1-k)_{2}, \ldots, e(n-1-k)_{j}$. We now define $U_{\ell}^{k}(v)=W_{1}^{k}(v)+\cdots+W_{j}^{k}(v) \subset f^{-1}\left(\mathcal{H}_{v}\right)$ and we claim that $U_{\ell}^{k}(v)$ is a cycle. Indeed,

$$
\begin{aligned}
\partial U_{\ell}^{k}(v) & =\partial\left(\sum_{i=1}^{j} W_{i}^{k}(v)\right)=\sum_{i=1}^{j} \partial W_{i}^{k}(v) \\
& =\sum_{i=1}^{j} U_{i}^{k-1}(v)=\sum_{\ell^{\prime}} W_{\ell^{\prime}}^{k-1}(v),
\end{aligned}
$$

where the chains $U_{i}^{k-1}(v)$ were constructed in the previous step in a similar manner and $\ell^{\prime}$ corresponds to the index of all $(n-k)$-cells that contains $v$.

The chain $U_{i}^{k-1}(v)$ is formed by looking at all the $(n-k)$-cells that contain $e(n-1-k)_{i}$ and hence will contain $e(n-2-k)_{\ell}$. Therefore, these $(n-k)$-cells can also be determined by looking at the edges of $\operatorname{Lk}\left(e(n-2-k)_{\ell}\right)$. Observe that for a fixed $i$, as we look at the chains of type $W_{\ell^{\prime}}^{k-1}(v)$ that comprise $U_{i}^{k-1}(v)$ we can alternatively look at the collection of edges in $\operatorname{Lk}\left(e(n-2-k)_{\ell}\right)$ that make up $U_{i}^{k-1}(v)$ and the chains $W_{\ell^{\prime}}^{k-1}(v)$ will be the vertices of such edges. However, because each edge contains exactly two vertices, as we do this for all $i$ each term in the last summation in (3.3) appears twice. Since our computation uses $\mathbb{Z}_{2}$ coefficients, we have (3.3) is zero establishing that $U_{\ell}^{k}(v)$ is a cycle.

Again, from the hypotheses that $f^{-1}\left(\mathcal{H}_{v}\right)$ is acyclic, we find a bounding $(k+1)$ chain $W_{\ell}^{k+1}(v) \subset f^{-1}\left(\mathcal{H}_{v}\right)$ of $U_{\ell}^{k}(v)$, that is, $\partial W_{\ell}^{k+1}(v)=U_{\ell}^{k}(v)$. Next, an analogous argument as in step 1 is used to find a neighborhood of $v$ in $\partial Y_{(n-2-k)}$ where the assignment of $W_{\ell}^{k+1}(v)$ is continuous for all points within it. This follows from the local diffeomorphism of $f$ and compactness if $W_{\ell}^{k+1}(v)$. This induces a cover of $\partial Y_{(n-2-k)}$ and by compactness we extract a finite subcover. Finally, we take finitely many barycentric subdivisions of $\partial Y$ until the mesh is smaller than the minimum diameter of the subcover.

Using the new triangulation, we repeat the assignment of the chain complexes in each of the previous steps 0 through $k$. In particular, this defines $W_{\ell}^{k+1}(v)$ for all points in $e(n-2-k) \ell$ and by the modification argument indicated above, we obtain a $(n-1)$-chain denoted by $W_{\ell}^{k+1} \times e(n-2-k)_{\ell}$ for each $\ell \in E(n-2-k)$.

Once we have completed all the $n-1$ steps, we put together the singular complexes constructed from the $(n-1)$-chains in each step by means of their attaching maps along their common boundary which will be explicitly computed. In order to finish the proof, we show that $\partial W^{n-1}=\partial X_{0}+\partial X_{1}$ and thus once we attach $X_{0}$ and $X_{1}$ to the boundary we will have the cycle $\Gamma^{n-1}$ we seek. 
We consider the decomposition of $W^{n-1}$ from the $(n-1)$-chains in each step, that is,

$$
W^{n-1}=\sum_{k=0}^{n-2} \sum_{\ell \in E(n-2-k)} W_{\ell}^{k+1} \times e(n-2-k)_{\ell} .
$$

For simplicity, let $S_{k}=\sum_{\ell \in E(n-2-k)} W_{\ell}^{k+1} \times e(n-2-k)_{\ell}$, then $\partial W^{n-1}=$ $\sum_{k=0}^{n-2} \partial S_{k}$. We now analyze each term separately. For $k=0$,

$$
\begin{aligned}
\partial S_{0} & =\left(\partial \sum_{\ell \in E(n-2)} W_{\ell}^{1} \times e(n-2)_{\ell}\right) \\
& =\sum_{\ell \in E(n-2)} \partial W_{\ell}^{1} \times e(n-2)_{\ell}+\sum_{\ell \in E(n-2)} W_{\ell}^{1} \times \partial e(n-2)_{\ell} \\
& =\sum_{\ell \in E(n-2)}\left(e(0)_{\ell}^{0}+e(0)_{\ell}^{1}\right) \times e(n-2)_{\ell}+\sum_{\ell \in E(n-2)} \sum_{j_{n-3}} W_{\ell}^{1} \times e(n-3)_{j_{n-3}} \\
& =\partial X_{0}+\partial X_{1}+\sum_{\ell \in E(n-2)} \sum_{j_{n-3}} W_{\ell}^{1} \times e(n-3)_{j_{n-3}},
\end{aligned}
$$

where $j_{n-3}$ denotes the index of all $(n-3)$-cells that belong to the boundary of $e(n-2)_{\ell}$. In general, for $0<k<n-2$,

$$
\begin{aligned}
\partial S_{k}= & \partial\left(\sum_{\ell \in E(n-2-k)} W_{\ell}^{k+1} \times e(n-2-k)_{\ell}\right) \\
= & \sum_{\ell \in E(n-2-k)} \partial W_{\ell}^{k+1} \times e(n-2-k)_{\ell}+\sum_{\ell \in E(n-2-k)} W_{\ell}^{k+1} \times \partial e(n-2-k)_{\ell} \\
= & \sum_{\ell \in E(n-2-k)} \sum_{j_{n-1-k}} W_{j_{n-1-k}}^{k} \times e(n-1-k)_{\ell} \\
& \quad+\sum_{\ell \in E(n-2-k)} \sum_{j_{n-3-k}} W_{\ell}^{k+1} \times e(n-3-k)_{j_{n-3-k}},
\end{aligned}
$$

where $j_{n-1-k}$ denotes the index of all $(n-1-k)$-cells that contain $e(n-2-k) \ell$ and $j_{n-3-k}$ denotes the index of all $(n-3-k)$-cells that belong to the boundary of $e(n-3-k)_{\ell}$. Finally, for $k=n-2$,

$$
\begin{aligned}
\partial S_{n-2} & =\partial\left(\sum_{\ell \in E(0)} W_{\ell}^{n-1} \times e(0)_{\ell}\right) \\
& =\sum_{\ell \in E(0)} \partial W_{\ell}^{n-1} \times e(0)_{\ell}+\sum_{\ell \in E(0)} W_{\ell}^{n-1} \times \partial e(0)_{\ell} \\
& =\sum_{\ell \in E(0)} \sum_{j_{1}} W_{j_{1}}^{n-2} \times e(0)_{\ell}+0,
\end{aligned}
$$


where $j_{1}$ denotes the index of all 1 -cells that contain $e(0)_{\ell}$ in its boundary.

Observe that the second summation term of $\partial S_{k}$ is the same as the first summation term of $S_{k+1}$ as we count each chain twice. Since we are using $\mathbb{Z}_{2}$-coefficients, (3.4) simplifies to $\partial W^{n-1}=\partial X_{0}+\partial X_{1}$.

Finally, from the construction of $W^{n-1}$ we see that for each $q \in \operatorname{supp} W^{n-1}$, $q \in f^{-1}\left(\mathcal{H}_{v}\right)$ for some $v \in \partial Y$. This concludes the proof of Lemma 3.4.

\section{Surjectivity}

In this section we consider the question of when a local diffeomorphism $f: M \rightarrow \mathbb{R}^{n}$ is surjective, based on the topology of the pre-images of hyperplanes. The trivial example of an inclusion map of the region between two planes satisfies Theorem 3.3 but it is not surjective. This indicates that further assumptions must be added. On the other hand, we are able to eliminate the homological assumption on the domain.

Theorem 4.1. Let $f: M \rightarrow \mathbb{R}^{n}$ be a local diffeomorphism where $M$ is a connected manifold. If the pre-image of every affine hyperplane is non-empty and acyclic, then $f$ is surjective.

The proof is based on geometric constructions involving foliation theory and the computation of linking numbers between certain singular chain complexes in the range $\mathbb{R}^{n}$. We remark that since the computation of linking numbers will occur in $\mathbb{R}^{n}$, it is not necessary to make any further assumptions on the homology groups of $M$. This is unlike the situation in Theorem 3.3, where we assumed $H_{n-1}(M)=0$.

Combining Theorem 4.1 and Theorem 3.3, we obtain the following characterization of $\mathbb{R}^{n}$, for $n \geq 2$.

Theorem 4.2. A smooth connected manifold $M$ is diffeomorphic to $\mathbb{R}^{n}$ if and only if $H_{n-1}(M)=0$ and there exists a local diffeomorphism $f: M \rightarrow \mathbb{R}^{n}$ such that the pre-image of every affine hyperplane is non-empty and acyclic.

4.1. Proof of Theorem 4.1. We establish surjectivity by showing that for each $R>0$ the ball of radius $R$ is fully contained in $f(M)$, that is, $\bar{B}(0 ; R) \subset f(M)$. Since translations do not change any of our hypotheses, let us assume that $0 \in f(M)$ and single out $o \in f^{-1}(0) \subset M$.

Next, from the local diffeomorphism assumption, there exists $\varepsilon>0$ such that $\bar{B}(0 ; \varepsilon) \subset f(M)$ and $f^{-1}(B(0 ; \varepsilon))$ has a diffeomorphic component $W^{n-1}(\varepsilon)$ which contains $o \in M$. Observe that for $R \leq \varepsilon$, we trivially have $\bar{B}(0 ; R) \subset f(M)$, so we restrict ourselves to the case $R>\varepsilon$.

We argue that we can find a way to expand $B(0 ; \varepsilon)$ within the image of $f$ so that it will contain a ball of radius any $R$. To this end, we shall choose directions 
for this expansion as follows. For $v \in S^{n-1}$, let $\mathcal{H}_{v}$ be the canonical codimension one foliation of $\mathbb{R}^{n}$ by hyperplanes orthogonal to $v$. Since the leaf space of $\mathcal{H}_{v}$ is homeomorphic to $\mathbb{R}$, we parameterize the leaves of $\mathcal{H}_{v}$ by $\mathcal{H}_{v}(t)$ where $t$ is the distance of the hyperplane $\mathcal{H}_{v}(t)$ to the origin. Because $\mathcal{H}_{v}(t)=\mathcal{H}_{-v}(-t)$, we will only consider $t \geq 0$. Let $\mathcal{N}_{v}=f^{*} \mathcal{H}_{v}$ be the pullback foliation of $M$ which, by definition, has the connected components of $f^{-1}\left(\mathcal{H}_{v}(t)\right)$ as leaves. Since our hypotheses states that the pre-images of hyperplanes are non-empty and connected, the leaf space of $\mathcal{N}_{v}$ is homeomorphic to $\mathbb{R}$ and we then write $\mathcal{N}_{v}(t)=f^{-1}\left(\mathcal{H}_{v}(t)\right)$. Next, we claim that for each $v \in S^{n-1}$, we may find a global transversal $\gamma_{v}$ to the foliation $\mathcal{N}_{v}$ that may be used to expand the image of $f$. More precisely, we have the following result.

Lemma 4.3. For each $u \in W^{n-1}(\varepsilon)$ with $f(u)=\varepsilon v, v \in S^{n-1}$, there exists a smooth path $\gamma_{v}:[0, \infty) \rightarrow M$ with $\gamma_{v} \pitchfork \mathcal{N}_{v}$ such that $f\left(\gamma_{v}(t)\right) \in \mathcal{H}_{v}(t)$ for $t \in[0, R]$.

The proof follows directly from transverse modification arguments in foliation theory (see [2]) and the fact that the leaves of $\mathcal{N}_{v}$ are non-empty and connected.

As we proceed with the proof of Theorem 4.1, let us fix a canonical identification of $S^{n-1}$ to $\partial B(0 ; \varepsilon)$ and $W^{n-1}(\varepsilon)$. By applying Lemma 4.3, we obtain directions $\gamma_{v}$ from which to expand $W^{n-1}(\varepsilon)$ up to $\gamma_{v} \cap \mathcal{N}_{v}(R)$. Then for a fixed $v$, we can locally modify $\gamma_{v}$ so that we can carry an entire neighborhood of $v$ in $W^{n-1}(\varepsilon)$ along $\gamma_{v}$ using the compactness of $\gamma_{v}([0, R])$. Repeating this process for each $v \in W^{n-1}(\varepsilon)$, we obtain a cover of $W^{n-1}(\varepsilon)$. However, because there is no canonical choice for $\gamma_{v}$, points belonging to the intersection of two neighborhoods may have multiply defined paths. Our approach will be similar to the one in Section 3. The key difference here is that we also need to control how each neighborhood is pushed along the global transversal $\gamma_{v}$.

Intuitively, we will push the cells of $W^{n-1}(\varepsilon)$ along $\gamma_{v}$ and possibly create broken pieces at each instant $t$. Using the hypotheses that $\mathcal{N}_{v}(t)$ is acyclic and $f$ is a diffeomorphism we will define bounding chains filling the gaps in each leaf. Furthermore, we will argue that these chain complexes constructed for $s, t$ will be homologous, hence we say that they are homologous relative to $t$. This process is depicted in Figure 4.1 and it is stated precisely in the lemma below.

Lemma 4.4. For each $R>0$, there exists a family of geometric (singular) chain complexes $W^{n-1}(t)$ that are homologous in $M \backslash\{0\}$ for $t \in(0, R]$ such that:

i) For $t \in(0, \varepsilon], W^{n-1}(t)=f^{-1}(\partial B(0 ; t))$.

ii) If $q \in \operatorname{supp} W^{n-1}(t)$, then $q \in \mathcal{N}_{v}(t)$ for some $v \in S^{n-1}$. 


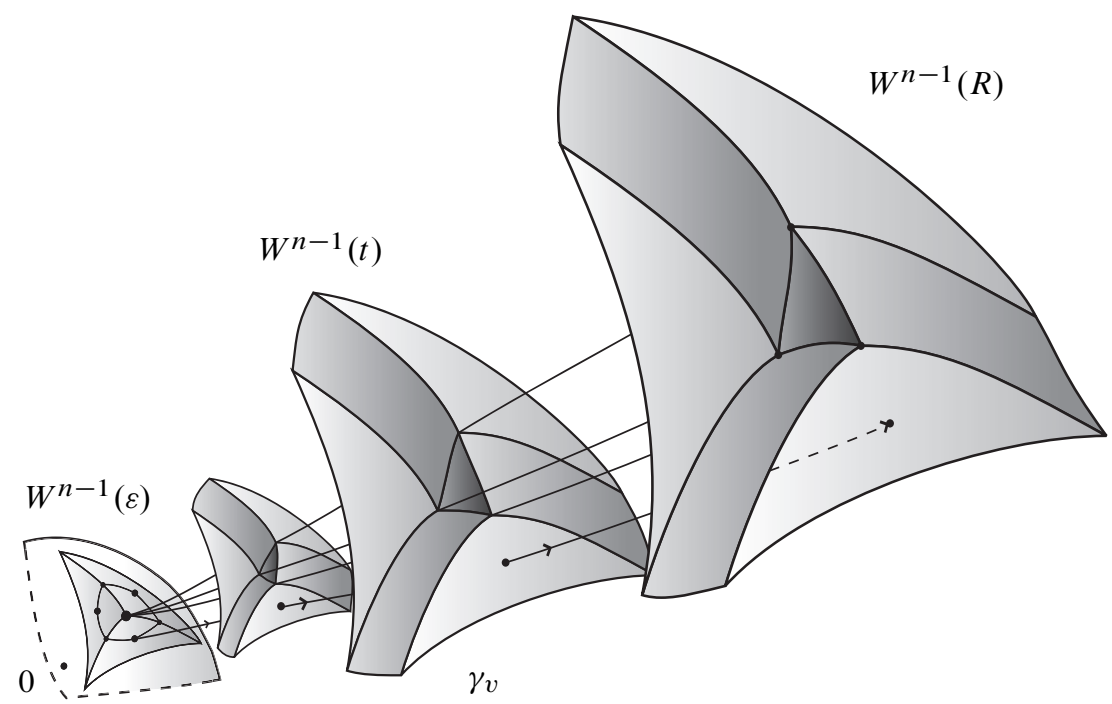

Figure 4.1. A local assemblage of chain complexes based on the triangulation of a sphere.

The proof of Lemma 4.4 uses combinatorial topology and foliation theory to explicitly construct such cycles. Since the process is lengthy and rather technical, we postpone it and continue with the proof of Theorem 4.1 .

Our strategy to show that $\bar{B}(0 ; R) \subset f(M)$ is by contradiction. Suppose there is $p \notin f(M)$. We compute the linking number between $p$ and $f\left(W^{n-1}(t)\right)=Z^{n-1}(t)$ in two ways, yielding different values. This argument is similar to standard reasoning in degree theory and is geometric in nature. As before, we work with $\mathbb{Z}_{2}$-coefficients.

Notice that since $f$ is continuous, we have that $Z^{n-1}(t)$ is a family of homologous cycles in $\mathbb{R}^{n} \backslash\{0\}$. Then for $t \in(0, \varepsilon], Z^{n-1}(t)=\partial B(0 ; t)$ and we have that the origin is contained in the inside of $Z^{n-1}(\varepsilon)$, more precisely, the linking number between the origin and $Z^{n-1}(\varepsilon)$ is equal to 1 .

From (ii) of Lemma 4.4, we have that $0 \notin Z^{n-1}(t)$ for each $t \in(0, R]$ and as mentioned above, $Z^{n-1}(t) \sim Z^{n-1}(R)$ in $\mathbb{R}^{n} \backslash\{0\}$. Therefore as intersection numbers, thus linking numbers are invariant under the same homology class, we have

$$
\operatorname{Lk}\left(Z^{n-1}(t), 0\right)=1 \text { for each } \varepsilon<t \leq R,
$$

where we consider the 0-normal cycle formed by the origin and a suitable point in the complement of a compact set containing $Z^{n-1}(t)$.

We claim that $p$ is inside $Z^{n-1}(R)$, that is, $\operatorname{Lk}\left(Z^{n-1}(R), p\right)=1$. Indeed, consider the segment $Y^{1}$ from 0 to $p$. We have that $Y^{1} \cap Z^{n-1}(R)=\emptyset$, otherwise 
it would imply $p \in f(M)$. By definition,

$$
\#\left(Z^{n-1}(R) \times Y^{1}\right)=\#\left(Y^{1} \times Z^{n-1}(R)\right)=0 .
$$

Computing the linking number between the cycle $Z^{n-1}(R)$ and $\partial Y^{1}$ using the fact that $\partial Y^{1}$ is a normal 0 -cycle, we have

$$
\begin{aligned}
0 & =\#\left(Y^{1} \times Z^{n-1}(R)\right)=\operatorname{Lk}\left(\partial Y^{1}, Z^{n-1}(R)\right)=\operatorname{Lk}\left(Z^{n-1}(R), \partial Y^{1}\right) \\
& =\operatorname{Lk}\left(Z^{n-1}(R), 0-p\right)=\operatorname{Lk}\left(Z^{n-1}(R), 0\right)-\operatorname{Lk}\left(Z^{n-1}(R), p\right) .
\end{aligned}
$$

Combining (4.3) and $\operatorname{Lk}\left(Z^{n-1}(R), 0\right)=1$ we obtain

$$
\operatorname{Lk}\left(Z^{n-1}(R), p\right)=1 .
$$

Observe that $Z^{n-1}(\varepsilon)=\partial B(0 ; \varepsilon)$, hence $\operatorname{Lk}\left(Z^{n-1}(\varepsilon), p\right)=0$. Finally, from the assumption that $p \notin f(M)$, we have $Z^{n-1}(R) \sim Z^{n-1}(\varepsilon)$ in $\mathbb{R}^{n}-\{p\}$ and again by the invariance of linking numbers on the homology class we obtain

$$
\operatorname{Lk}\left(Z^{n-1}(R), p\right)=\operatorname{Lk}\left(Z^{n-1}(\varepsilon), p\right)=0 .
$$

This is a contradiction, therefore it must be the case that $p \in f(M)$ and hence $f$ is surjective.

4.2. The construction of a family of homologous cycles. We now complete the proof of Theorem 4.1 by establishing the technical proof of Lemma 4.4. We employ a similar technique as in Section 3, that is, we use triangulations as a tool to keep track of intersections in the coverings. For simplicity, let $W^{n-1}(\varepsilon)=W$ and consider a triangulation of $W$ with cells $e(k)_{\ell} ; k=0, \ldots, n-1$ and $\ell \in E(k) \subset \mathbb{N}$ where $E(k)$ is the set of indexes of all the $k$-cells in $W$.

The idea of the construction of $W^{n-1}(t)$ is in essence geometric, and can be outlined as follows. Consider a triangulation with sufficiently small mesh. For each top dimensional cell of $W$, we push it along a global transversal $\gamma_{v}$ emanating from one of its points up to the level $R$ and use the local modification of $\gamma_{v}$ for points within the cell to push these points. The key issue is that a point $v$ belonging to the boundary of a top dimensional cell may be pushed along multiple choices of $\gamma_{v}$, one for each top dimensional cell it belongs to. Hence the $W^{n-1}(t)$ may not be well defined; geometrically, this will create broken pieces at each level. However, by considering the collections of cells that contain $v$, via the link of $v$, we will show that for each $t$, the multiply defined chain complexes form a cycle in the pre-image of $\mathcal{H}_{v}(t)$. Thus by acyclicity, we can fill these gaps with bounding chains. Furthermore, the process will be done so it is homologous relative to $t$, that is, as we consider different chain complexes for each $t$. Now, as we begin to formally describe $W^{n-1}(t)$, we will do so in steps enumerated from 0 to $n-1$, outlined below. 
Step 0. For each point $v \in W$, suppose $v \in e(n-1)_{\ell}$ for some $\ell \in E(n-1)$. From Lemma 4.3 we obtain a global transversal $\gamma_{v}$ to the foliation $\mathcal{N}_{v}$. We then define the following 0-chains, that is, points where $\gamma_{v}$ intersect the leaves of $\mathcal{N}_{v}$. Let $W_{\ell}^{0}(v, t)=\gamma_{v}(t) \in \mathcal{N}_{v}(t)$ for $t \in[\varepsilon, R]$. By compactness of $\gamma_{v}([0, R])$ and the fact that $f$ is a local diffeomorphism, there is a neighborhood $\mathcal{V}_{v} \subset M$ of $\gamma_{v}([0, R])$ such that we can continuously modify $\gamma_{v}$ to obtain a global transversal $\gamma_{v^{\prime}}$ for all $v^{\prime}$ in a neighborhood $\mathcal{O}_{v} \subset W$ of $v$, as depicted in Figure 4.2.

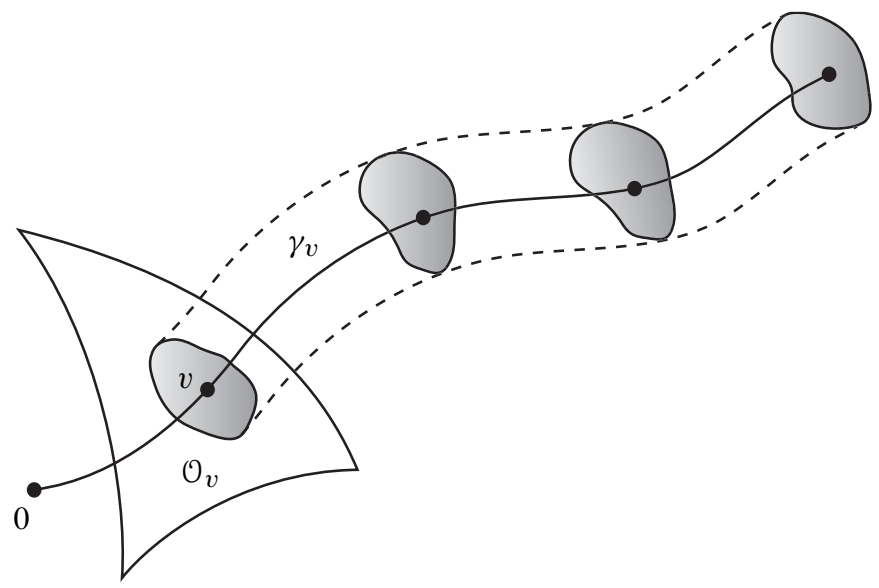

Figure 4.2. Local modification of global transversals.

Then, by the compactness of $W$, we obtain a finite subcover of $W$ from $\left\{\mathcal{O}_{v}\right\}$. Now with such subcover, we iterate finitely many barycentric subdivisions of $W$ until its mesh is smaller than the minimum diameter of the subcover. In this process, we obtain a new triangulation of $W$ with the property that, for each $v \in e(n-1)_{\ell}$, we may continuously define $\gamma_{v}$ for all points in $e(n-1)_{\ell}$. In fact, we define, for each $t \in[\varepsilon, R]$ and $\ell \in E(n-1)$, a $(n-1)$-chain denoted by $W_{\ell}^{0}(t) \times e(n-1)_{\ell}$ which is topologically equivalent to $W_{\ell}^{0}(v, t) \times e(n-1)_{\ell}$ and varies continuously on $t$.

Step 1. Consider points $v$ in the $(n-2)$-skeleton of $W$. Suppose $v \in e(n-2)_{\ell}$ for some $\ell \in E(n-2)$. Then $v$ belongs to the intersection of two $(n-1)$-cells that can be determined by looking at the vertices in $\operatorname{Lk}\left(e(n-2)_{\ell}\right)$. Without loss of generality let $v \in e(n-1)_{1} \cap e(n-1)_{2}$. Then for each $t \in[\varepsilon, R]$, we have defined in the previous step the points $W_{1}^{0}(v, t), W_{2}^{0}(v, t) \in \mathcal{N}_{v}(t)$ along path emanating from each top dimensional cell. Now we can join such points by a path $W_{\ell}^{1}(v, t)$ lying in $\mathcal{N}_{v}(t)$ as it is acyclic.

Once we construct $W_{\ell}^{1}(v, t)$, we claim that it can be locally modified for all 
points in a neighborhood of $(v, t)$ in $W_{(n-2)} \times[\varepsilon, R]$. Indeed, for each $v \in W_{(n-2)}$ and $t \in[\varepsilon, R]$, the path $W_{\ell}^{1}(v, t)$ is compact and hence we may find a neighborhood $\mathcal{U}(v, t) \subset M$ of $W_{\ell}^{1}(v, t)$ such that the (local) gradient flow of the height function $f_{v}: M \rightarrow \mathbb{R}^{n}$ given by $f_{v}(x)=\langle f(x), v\rangle$ can be used to continuously define $W_{\ell}^{1}(v, t)$ for nearby $t$. Also, the fact that $f$ is a local diffeomorphism continuously defines $W_{\ell}^{1}(v, t)$ for all nearby $v$ in $W_{(n-2)}$.

The process above provides a cover $\{\mathcal{U}(v, t)\}$ of $W_{(n-2)} \times[\varepsilon, R]$. By compactness, we may find a finite subcover which induces a cover of $W$. Indeed, in step 0 each top dimensional cell is pushed diffeomorphically along the global transversal $\gamma_{v}$. We can now iterate finitely many barycentric subdivisions of $W$ so its mesh is smaller than the minimum diameter of the subcover above restricted to $W$. Next, we repeat all the constructions up to this point using the new triangulation. Observer that this guarantees that each cell $e(n-2)_{\ell}$ is contained in a member of the finite subcover.

Now let us consider a partition of $[\varepsilon, R]$ induced by this subcover, that is, we have $\varepsilon=t_{0}<t_{1}<\cdots<t_{N}=R$ for some $N \in \mathbb{N}$. From the choice of subdivision we can continuously modify $W_{\ell}^{1}(v, t)$ for $t \in\left(t_{i}, t_{i+1}\right)$ and $v \in e(n-2)_{\ell}$ by the argument above. The key problem is that for the endpoint we may possibly have two chains defined, each coming from the adjacent intervals. However, the fact that each leaf of $\mathcal{N}_{v}$ is acyclic yields a similar construction as the transverse modification method (see [2]) to ensure that whenever ambiguity occurs, the choice will be homologous relative to $t$.

The details are as follows. For each $i=i, \ldots, N-1$, let the two choices for a bounding chain $W_{\ell}^{1}\left(v, t_{i}\right)$ be $W_{\ell}^{1}\left(v, t_{i}\right)^{-}$and $W_{\ell}^{1}\left(v, t_{i}\right)^{+}$, where $W_{\ell}^{1}\left(v, t_{i}\right)^{-}$is the chain defined continuously from $W_{\ell}^{1}(v, t), t \in\left(t_{i-1}, t_{i}\right)$ and the second one, $W_{\ell}^{1}\left(v, t_{i}\right)^{+}$, is the chain defined continuously from $W_{\ell}^{1}(v, t), t \in\left(t_{i}, t_{i+1}\right)$. By default, we agree to always choose $W_{\ell}^{1}\left(v, t_{i}\right)^{+}$. This will not be ambiguous because we can choose either chain complex. Indeed, $W_{\ell}^{1}\left(v, t_{i}\right)^{-} \sim W_{\ell}^{1}\left(v, t_{i}\right)^{+}$since from construction they have the same boundary and, by acyclicity of $\mathcal{N}_{v}\left(t_{i}\right)$, there is a bounding chain contained in $\mathcal{N}_{v}\left(t_{i}\right)$. Finally, we must consider a new neighborhood $\widetilde{\mathcal{U}}\left(v, t_{i}\right)$ of such bounding chain where the restriction of $f$ is a diffeomorphism. Doing so for every $v \in W_{(n-2)}$ and $i=1, \ldots, N$ we obtain a new cover of $W$ by adding the collection of sets $\tilde{\mathcal{U}}\left(v, t_{i}\right)$ to the finite subcover considered up to this point. This is done to ensure that the process will always yield chains homologous relative to $t$. Then iterate finitely many barycentric subdivisions of $W$ to obtain a triangulation with mesh sufficiently small to define chains $W_{\ell}^{1}(v, t)$ continuously for all points $v \in e(n-2)_{\ell}$ for each $\ell$ and by construction these chains are homologous relative to $t$ with continuously varying bounding chains.

This adaptation of the transverse modification argument produces for each $t \in$ $[\varepsilon, R]$ and $\ell \in E(n-2)$, a $(n-1)$-chain complex denoted by $W_{\ell}^{1}(t) \times e(n-2)_{\ell}$ which is topologically equivalent to $W_{\ell}^{1}(v, t) \times e(n-2)_{\ell}, v \in e(n-2)_{\ell}$, and is homologous relative to $t$. This concludes step 1 . 
Now we give the general procedure for $1<k \leq n-1$.

Step $k$ : Consider points in the $(n-1-k)$-skeleton of $W$. Suppose $v \in e(n-1-k)_{\ell}$ for some $\ell \in E(n-1-k)$. We are interested in identifying all the $(n-k)$-cells that contain $e(n-1-k)_{\ell}$ in its boundary, i.e., that contain $v$. This can be accomplished by looking at the vertices of $\operatorname{Lk}(e(n-1-k) \ell)$. for simplicity, suppose that those are $e(n-k)_{1}, e(n-k)_{2}, \ldots, e(n-k)_{m}$ for some $m \in \mathbb{N}$. For each $t \in[\varepsilon, R]$, consider the $(k-1)$-chain

$$
W_{1}^{k-1}(v, t)+\cdots+W_{m}^{k-1}(v, t),
$$

where the chains $W_{j}^{k-1}(v, t)$ were constructed in step $k-1$. We claim that the $(k-1)$-chain in (4.6) is a cycle. Indeed,

$$
\partial\left(\sum_{j=1}^{m} W_{j}^{k-1}(v, t)\right)=\sum_{j=1}^{m} \partial W_{j}^{k-1}(v, t)=\sum_{j=1}^{m}\left(\sum_{\ell^{\prime}} W_{\ell^{\prime}}^{k-2}(v, t)\right),
$$

where $\ell^{\prime}$ corresponds to the index of all $(n-k+1)$-cells in $W$ that contain $e(n-1-k) \ell$. Now the argument is completely analogous to the one given in the injectivity case, i.e, it follows from the observation that an edge contains exactly two vertices. Since $\mathcal{N}_{v}(t)$ is acyclic, there exists a $k$-chain $W_{\ell}^{k}(v, t)$ that bounds $\sum_{j=1}^{m} W_{j}^{k-1}(v, t)$.

We now argue that the chain $W_{\ell}^{k}(v, t)$ may be continuously modified with respect to $v$ and within the same homology class relative to $t$. This is similar to the construction as in step 1, except that we repeat it for each interval of the partition obtained in step $k-1$, so we omit the details. Finally, for each $t \in[\varepsilon, R]$ and $\ell \in E(n-1-k)$, we obtain a $(n-1)$-chain denoted by $W_{\ell}^{k}(t) \times e(n-1-k)_{\ell}$ which for $v \in e(n-1-k)_{\ell}$, is topologically equivalent to $W_{\ell}^{k}(v, t) \times e(n-1-k)_{\ell}$, and is homologous relative to the parameter $t$.

Remark 4.1. In the last step $n-1$, we consider the 0 -skeleton of $W$ which is discrete, hence no further subdivisions are necessary.

Once all $n$ steps are completed, for each $t \in[\varepsilon, R]$, we put together all the constructed chain complexes via the obvious attaching maps based on the intersection of the cells in $W$ as indicated by their construction in each step. This defines $W^{n-1}(t)$ as follows:

$$
W^{n-1}(t)=\sum_{k=0}^{n-1} \sum_{\ell \in E(n-1-k)} W_{\ell}^{k}(t) \times e(n-1-k)_{\ell}
$$

We observe that $W^{n-1}(t) \sim W^{n-1}(s)$ in $M \backslash\{o\}$ for $t, s \in[\varepsilon, R]$. Indeed, by construction if $t, s \in\left(t_{i-1}, t_{i}\right)$ for $i=1, \ldots, N$, we can use the local gradient flow of the corresponding height functions to continuously modify the chain $W^{n-1}(t)$ to 
$W^{n-1}(s)$, each chain $W_{\ell}^{n-1-k}(t) \times e(k)_{\ell}$ at a time. Otherwise, the only problem is at the end points $t_{i}$, where again by construction, the chains of $W^{n-1}\left(t_{i}\right)$ are homologous in $\mathcal{N}\left(t_{i}\right)=\left\{p \in M \mid p \in \mathcal{N}_{v}\left(t_{i}\right)\right.$ for some $\left.v \in S^{n-1}\right\}$, thus ensuring that $W^{n-1}(t)$ are homologous in $M \backslash\{0\}$.

It now remains to show that $W^{n-1}(t)$ is a cycle in $M$. The computation below is quite similar to the one done in Section 3. For simplicity, let $S_{k}(t)=$ $\sum_{\ell \in E(n-1-k)} W_{\ell}^{k}(t) \times e(n-1-k)_{\ell}$, so that $\partial W^{n-1}(t)=\sum_{k=0}^{n-1} \partial S_{k}(t)$.

Considering each term separately, we have for $k=0$,

$$
\begin{aligned}
\partial S_{0}(t) & =\partial\left(\sum_{\ell \in E(n-1)} W_{\ell}^{0}(t) \times e(n-1)_{\ell}\right) \\
& =\sum_{\ell \in E(n-1)} \partial W_{\ell}^{0}(t) \times e(n-1)_{\ell}+\sum_{\ell \in E(n-1)} W_{\ell}^{0}(t) \times \partial e(n-1)_{\ell} \\
& =0+\sum_{\ell \in E(n-1)} \sum_{j_{n-2}} W_{\ell}^{0} \times e(n-2)_{j_{n-2}},
\end{aligned}
$$

where $j_{n-2}$ denotes the index of all $(n-2)$-cells that belong to the boundary of $e(n-1)_{\ell}$. In general, for $1<k<n-1$,

$$
\begin{aligned}
\partial S_{k}(t)= & \partial\left(\sum_{\ell \in E(n-1-k)} W_{\ell}^{k}(t) \times e(n-1-k)_{\ell}\right) \\
= & \sum_{\ell \in E(n-1-k)} \partial W_{\ell}^{k}(t) \times e(n-1-k)_{\ell} \\
& \quad+\sum_{\ell \in E(n-1-k)} W_{\ell}^{k}(t) \times \partial e(n-1-k)_{\ell} \\
= & \sum_{\ell \in E(n-1-k)} \sum_{j_{n-k}} W_{j_{n-k}}^{k-1}(t) \times e(n-1-k)_{\ell} \\
& \quad+\sum_{\ell \in E(n-1-k)} \sum_{j_{n-2-k}} W_{\ell}^{k}(t) \times e(n-2-k)_{j_{n-2-k}},
\end{aligned}
$$

where $j_{n-k}$ denotes the index of all $(n-k)$-cells that contain $e(n-1-k)_{\ell}$ and $j_{n-2-k}$ denotes the index of all $(n-2-k)$-cells that belong to the boundary of $e(n-1-k)_{\ell}$. Finally, for $k=n-1$,

$$
\begin{aligned}
\partial S_{n-1}(t) & =\partial\left(\sum_{\ell \in E(0)} W_{\ell}^{n-1}(t) \times e(0)_{\ell}\right) \\
& =\sum_{\ell \in E(0)} \partial W_{\ell}^{n-1}(t) \times e(0)_{\ell}+\sum_{\ell \in E(0)} W_{\ell}^{n-1}(t) \times \partial e(0)_{\ell} \\
& =\sum_{\ell \in E(0)} \sum_{j_{1}} W_{j_{1}}^{n-2}(t) \times e(0)_{\ell}+0,
\end{aligned}
$$


where $j_{1}$ denotes the index of all 1-cells that contain $e(0)_{\ell}$ in its boundary.

As we sum the terms in $W^{n-1}(t)$, we see that the second summation term appearing in $\partial S_{k}(t)$ coincided with the first summation in $\partial S_{k+1}(t)$. Indeed, these terms count the same objects twice, and hence we obtain zero (recall that we are working with $Z_{2}$ coefficients). Therefore $W^{n-1}(t)$ is a cycle and this finishes the proof of Lemma 4.4.

\section{Final remarks}

Having obtained independent results on injectivity and surjectivity, we note that our main result follows from Theorem 3.3 and Theorem 4.1. Recall

Theorem 1.1. A local diffeomorphism $f: \mathbb{R}^{n} \rightarrow \mathbb{R}^{n}$ is bijective if and only if the pre-image of every affine hyperplane is non-empty and acyclic.

We also have the following analytic condition that establishes whether a local diffeomorphism is bijective. Given a complete Riemannian metric $g$ on $\mathbb{R}^{n}$ and a smooth function $h: \mathbb{R}^{n} \rightarrow \mathbb{R}$, the gradient of $h$ relative to $g$, denoted by $\nabla^{g} h$, satisfies $g_{x}\left(\nabla^{g} h, w\right)=d h_{x}(w)$ for all $w \in \mathbb{R}^{n}$. Our analytic result is the following.

Corollary 5.1. A local diffeomorphism $f: \mathbb{R}^{n} \rightarrow \mathbb{R}^{n}$ is bijective if for each $v \in$ $S^{n-1}$, there exists a complete metric $g_{v}$ on $\mathbb{R}^{n}$ such that

$$
\inf _{x \in \mathbb{R}^{n}}\left|\nabla^{g_{v}} f_{v}(x)\right|_{g_{v}}>0 .
$$

It is easy to see that such condition implies that the pre-images of hyperplanes are acyclic, hence the result follows. We compare this result with the work in [7] where we can now choose the metric to suit the unit vector. Finally, We can also state an analytical result implying only injectivity.

Corollary 5.2. A local diffeomorphism $f: \mathbb{R}^{n} \rightarrow \mathbb{R}^{n}$ is injective provided there exists $w \in S^{n-1}$ with the property that for each unit vector $v$ perpendicular to $w$, there exists a complete Riemannian metric $g_{v}$ on $\mathbb{R}^{n}$ such that

$$
\inf _{x \in \mathbb{R}^{n}}\left\|D f(x)^{*} v\right\|_{g_{v}}>0 .
$$

Observe that in our injectivity results we did not need topological hypotheses on the pre-image of every hyperplanes, hence the result holds.

\section{References}

[1] E. C. Balreira, Incompressibility and global inversion. Topol. Methods Nonlinear Anal., to appear. 
[2] A. Candel and L. Conlon, Foliations I. Grad. Stud. Math. 23, Amer. Math. Soc., Providence, R.I., 2000. Zbl 0936.57001 MR 1732868

[3] E. Connell H. Bass and D. Wright, The Jacobian conjecture: reduction of degree and formal expansion of the inverse. Bull. Amer. Math. Scoc. 7 (1982), 287-330. Zbl 0539.13012 MR 0663785

[4] J. Hadamard, Sur les transformations ponctuelles. Bull. Soc. Math. France 34 (1906), 71-84. JFM 37.0672.02 MR 1504541

[5] L. Nirenberg, Topics in nonlinear functional analysis. Courant Lect. Notes Math. 6, New York University, Courant Institute of Mathematical Sciences, New York 2001. Zbl 0992.47023 MR 1850453

[6] S. Nollet, L. Taylor, and F. Xavier, Birationality of étale maps via surgery. J. Reine Angew. Math. 627 (2009), 83-95. Zbl 1161.14042 MR 2494914

[7] S. Nollet and F. Xavier, Global inversion via the Palais-Smale condition. Discrete Contin. Dyn. Syst. 8 (2002), 17-28. Zbl 1008.58009 MR 1877825

[8] — Holomorphic injectivity and the Hopf map. Geom. Funct. Anal. 14 (2004), no. 6, 1339-1351. Zbl 1073.32005 MR 2135170

[9] —, On Kulikov's problem. Arch. Math. 89 (5) (2007), 385-389 Zbl 1135.14049 MR 2363688

[10] C. Palmeira, Open manifolds foliated by planes. Ann. of Math. 107 (1978), 109-131. Zbl 0382.57010 MR 0501018

[11] R. Plastock, Homeomorphisms between Banach spaces. Trans. Amer. Math. Soc. 200 (1974), 169-183. Zbl 0291.54009 MR 0356122

[12] P. J. Rabier, Ehresmann fibrations and Palais-Smale conditions for morphisms of Finsler manifolds. Ann. of Math. 146 (1997), no. 2, 647-691. Zbl 0919.58003 MR 1491449

[13] A. Schinzel, Polynomials with special regard to reducibility. In Encyclopedia Math. Appl. 77, Cambridge University Press, Cambridge 2000. Zbl 0956.12001 MR 1770638

[14] Jacob T. Schwartz, Differential geometry and topology, Gordon and Breach, New York 1968. Zbl 0187.45006

[15] B. Smyth and F. Xavier, Injectivity of local diffeomorphisms from nearly spectral conditions. J. Differential Equations 130 (1996), 406-414. Zbl 0870.58036 MR 1410896

[16] Arno van den Essen, Polynomial automorphisms and the Jacobian conjecture. Progr. Math. 190, Birkhäuser, Basel 2000. Zbl 0962.14037 MR 1790619

[17] J. H. C. Whitehead, On $C^{1}$-complexes. Ann. of Math. (2) 41 (1940), 809-824. Zbl 0025.09203 MR 0002545

[18] F. Xavier, Injectivity as a transversality phenomenon in geometries of negative curvature. Illinois J. Math. 43 (2) (1999), 256-263. Zbl MR

[19] _ - Using Gauss maps to detect intersections. L'Enseign. Math. 53 (2007), 15-31. Zbl 05230194 MR 2343343

[20] —, Rigidity of the identity. Commun. Contemp. Math. 9 (5) (2007), 691-699. Zbl 1141.32004 MR 2361737

[21] _ - The global inversion problem: a confluence of many mathematical topics. Mat. Contemp., to appear. 
Vol. 85 (2010)

Received January 23, 2008

Eduardo Cabral Balreira, Department of Mathematics, Trinity University, San Antonio, TX 78212, U.S.A.

E-mail: ebalreir@trinity.edu 